\title{
Clima organizacional: análise multivariada da percepção dos funcio- nários técnico-administrativos da Universidade Federal de Alagoas
}

\author{
Organizational climate: multivariate analysis of Universidade Federal de Alagoas technical- \\ administrative employees perception
}

\author{
Rogerio Lira Silva \\ Mestre em Administração Pública \\ Universidade Federal de Alagoas \\ rogerioliras@gmail.com \\ Luciana Peixoto Santa Rita \\ Doutora em Administração \\ Universidade Federal de Alagoas \\ upsantarita@gmail.com
}

\section{Resumo}

Este trabalho tem como objetivo analisar o clima organizacional a partir da percepção dos funcionários técnico-administrativos da Universidade Federal de Alagoas (UFAL). Metodologicamente, esta pesquisa foi classificada como descritiva, com abordagem quantitativa, e a coleta de dados foi realizada por meio de levantamento ou survey. Os dados foram coletados nos meses de outubro a dezembro de 2018 , em todos os campi da UFAL, por meio eletrônico. A amostra não-probabilística foi definida em uma população de 1.246 funcionários, com margem de erro de $4,84 \%$ e nível de confiança em $95 \%$. Nos resultados, a avaliação dos fatores e categorias que possuem importantes reflexos sobre o construto clima organizacional é predominantemente positiva. Nas conclusões, os dados permitiram a identificação do índice do clima organizacional positivo, das categorias imagem e avaliação institucional, desenvolvimento de recursos humanos, benefícios e incentivos, organização e condições de trabalho, relacionamento interpessoal, sucessão político-administrativa e satisfação pessoal.

\section{Palavras-chave}

Clima organizacional. Instituições de Ensino Superior. Análise Multivariada. Survey. Universidade Federal de Alagoas.

\begin{abstract}
This work aims to analyze the organizational climate from the perception of technical-administrative employees of Universidade Federal de Alagoas (UFAL). This research was classified as descriptive with a quantitative approach and data collection was carried out by survey. The data collection was performed electronically from October to December 2018 on all UFAL campuses. The non-probabilistic sample was defined in a population of 1,246 employees with $4.84 \%$ margin of error and confidence level of $95 \%$. The results showed that evaluation of the factors and categories with important reflections on the organizational climate construct was predominantly positive. The conclusion data allowed the identification of the positive organizational climate index and the categories image and institutional evaluation, development of human resources, benefits and incentives, organization and working conditions, interpersonal relationships, political-administrative succession and personal satisfaction.
\end{abstract}

\section{Keywords}

Organizational climate. Higher education institutions. Multivariate analysis. Survey. Universidade Federal de Alagoas. 


\section{INTRODUÇÃO}

Sabe-se que a principal fonte de mudança que atinge diretamente as organizações é resultado de reflexos da internacionalização das atividades em busca de uma economia global, ou seja, da globalização (GRIFFIN; MOORHEAD, 2016). Muito embora seja crescente a automação das atividades nas organizações, provocada, em especial, pela globalização, as pessoas continuam sendo um fator determinante para o crescimento das instituições. Nesse sentido, vê-se na literatura que é importante que a Gestão de Recursos Humanos (GRH) das organizações, sejam elas públicas ou privadas, proporcione um ambiente de trabalho adequado para seus funcionários (COSTA; TANIGUCHI, 2009; ARABAC, 2010; NATÁRIO; ARAÚJO; COUTO, 2014; MORO et al., 2015; CURVO; HEINZMANN, 2017).

A atmosfera do ambiente de trabalho repercute no contentamento ou descontentamento dos funcionários e pode significar importantes reflexos na produtividade, eficiência e eficácia da organização (SCHNEIDER; EHRHART; MACEY, 2013). A fim de identificar as principais variáveis que influenciam a produtividade e o bem-estar dos funcionários, diversos estudiosos, como Litwin e Stringer (1968), Campbell e Stanley (1970), Schneider (1975), La Follete e Sims (1975), Kolb, Rubin e McIntyre (1978), Sbragia (1983), Coda (1997), Tachizawa e Andrade (1999), Salgado Neto (2001), Rizzatti (2002), Luz (2003) e Silva (2003), dedicaramse ao estudo do clima organizacional.

Para esses autores, os fatores de (des)contentamento podem ser mensurados pela análise do clima organizacional, que, atualmente, tem sido utilizado como ferramenta pelas organizações que buscam promover o bem-estar dos seus funcionários. Por isso, as organizações que apresentam excelentes resultados nas pesquisas de clima organizacional tornamse referência em ambientes adequados para o trabalho. Esse bem-estar tem impactado positivamente na produtividade do local (SCHNEIDER; EHRHART; MACEY, 2013; LIMA; AMORIM; FISCHER, 2015; BARROS; MELO, 2016).

A despeito de a pesquisa de clima organizacional ser um tema consideravelmente discutido, as primeiras delas tiveram origem nos Estados Unidos, na década de 1960, com os trabalhos realizados por Forehand e Gilmer (1964), que se debruçaram sobre problemas relacionados à conceituação e à mensuração do clima organizacional. Já no Brasil, tal tipo de pesquisa começou a ser realizada em 1974, com o trabalho de Saldanha (1974), que envolvia o estudo da atmosfera organizacional e o bem-estar psíquico dos trabalhadores (PEREIRA; PEREIRA, 2014; REGIS FILHO, 2015; ZAMBRANO CAMPOVERDE; RAMÓN PINEDA; ESPINOZAFREIRE, 2017).

O estudo do clima organizacional em Instituições de Ensino Superior (IES) teve início nos anos 1980, por Moran e Volkwein (1988). Esse dado sinaliza a preocupação das IES que, até então, não tinham um olhar voltado para o clima, mesmo com características tão distintas de outras organizações que, per se, já justificariam a necessidade de conhecer a percepção de seus funcionários (SANTOS; VASQUEZ, 2012; VELA; GARRIDO; PÉREZ, 2016).

Com isso, este trabalho teve como objetivo geral analisar a percepção do clima organizacional da Universidade Federal de Alagoas (UFAL) sob a ótica dos funcionários técnicoadministrativos. Já os objetivos específicos foram verificar qual é o índice de satisfação dos funcionários técnico-administrativos da UFAL; e, identificar os agrupamentos e as correlações mais expressivos entre os fatores e as categorias do modelo de Rizzatti (1995) que envolve a análise de seis categorias: a imagem da organização; o desenvolvimento de recursos 
humanos; a organização e condições de trabalho; o relacionamento interpessoal; a sucessão político-administrativa; e, a satisfação pessoal.

Sendo assim, esta pesquisa surge da necessidade de buscar respostas para o seguinte questionamento: como é percebido o clima organizacional na UFAL a partir da ótica dos funcionários técnico-administrativos?

Justifica-se este estudo, em termos científicos, pela importância de se analisar o clima organizacional nas IES, a partir da identificação de variáveis que, direta ou indiretamente, influenciam no bem-estar dos funcionários e, em especial, no desempenho e produtividade nas IES, apresentando a pesquisa de clima organizacional como ferramenta de suporte à gestão de recursos humanos. Almeja-se também contribuir com o aprofundamento dos conhecimentos teóricos a partir das constatações encontradas na pesquisa empírica do clima organizacional.

\section{FUNDAMENTAÇÃO TEÓRICA}

A primeira pesquisa relacionada ao clima organizacional data de 1927, e é conhecida como experimento de Hawthorne, no qual Elton Mayo buscou estabelecer relação entre as condições físicas do ambiente de trabalho e a produtividade. Especificamente, ele analisou a influência da iluminação e constatou que ela poderia se relacionar com os níveis de produtividade dos empregados da fábrica Western Electric Company. No entanto, após diversos experimentos, Mayo observa que a produtividade se eleva com a melhoria das condições físicas, mas ao passo em que as condições físicas são restabelecidas à condição inicial, a produtividade se matinha em alto patamar. Com isso ele conclui que a produtividade estava diretamente relacionada, não a fatores físicos, mas a fatores relacionados ao comportamento, em especial, porque os funcionários percebiam que seus superiores demonstravam preocupação com as condições de trabalho e com os trabalhadores (MENEZES; GOMES, 2010).

Já em 1939, outros estudos foram realizados, dessa vez conduzidos por Lewin, Lippitt e White, na tentativa de investigar as condições psicológicas ou o comportamento dos líderes que afetam os liderados. Nesses estudos, os pesquisadores deram destaque à investigação de elementos culturais que poderiam apresentar explicações sobre os ambientes sociais, a exemplo dos ambientes organizacionais (MENEZES; GOMES, 2010; KINPARA; LAROS, 2014).

No Brasil, o primeiro trabalho relacionado ao tema foi realizado por Saldanha, em 1974. Nesse trabalho, Saldanha ressalta a importância do bem-estar psíquico dos indivíduos para que as organizações alcancem seus objetivos. Seguido por Saldanha, em 1977, Souza (1978) estabeleceu uma comparação entre o clima organizacional de uma empresa privada, um órgão público e uma universidade (REGIS FILHO, 2015).

Litwin e Stringer (1968) definem clima organizacional como sendo um conjunto de propriedades mensuráveis do ambiente de trabalho, percebidas, direta ou indiretamente, pelos indivíduos que vivem e trabalham neste ambiente e que influencia a motivação e o comportamento dessas pessoas (LITWIN, STRINGER, 1968; SOUZA, 1978). Já Luz (1995) entende que "clima organizacional é o reflexo do estado de espírito ou de ânimo das pessoas, que predomina numa organização, em um determinado período" (LUZ, 1995, p. 6).

Por sua vez, Coda (1993) afirma que clima é o indicador do grau de satisfação dos membros de uma empresa, em relação a diferentes aspectos da cultura ou realidade aparente da organização, tais como políticas de recursos humanos, modelo de gestão, missão, processo de comunicação, valorização profissional e identificação com a empresa (CODA, 
1993). Os autores Schneider e Barbera (2014) definem clima organizacional como sendo o significado que os funcionários atribuem às políticas, práticas e procedimentos das organizações.

Somando-se aos conceitos apresentados, Rizzatti (2002, p. 27), por sua vez, entende que clima é:

\begin{abstract}
Um conceito importante para descrever as percepções dos indivíduos sobre as organizações em que trabalham. Por se tratar de um conceito importante e abrangente, consegue resumir numerosas percepções num pequeno grupo de dimensões para caracterizar as percepções das pessoas sobre os grupos e equipes em que trabalham, bem como para descrever sistemas sociais, tais como famílias, clubes, instituições privadas e públicas. Por outro lado, o clima representa uma soma das expectativas geradas em uma situação, e é um fenômeno grupal, resultante e característico de uma coletividade e um conceito que engloba tanto os fatos humanos e materiais como os abstratos, resultantes do convívio humano institucional e da cultura presente na instituição.
\end{abstract}

O conceito de Rizzatti (2002) envolve a percepção dos funcionários sintetizadas em dimensões. $O$ autor não se omite em associar o conceito às características da satisfação e motivação e destaca que a pesquisa de clima organizacional se apresenta como um importante instrumento de feedback dos funcionários que podem ser utilizados pela gestão para direcionar suas estratégias organizacionais (RIZZATTI, 2002).

Vários estudiosos já se debruçaram sobre a investigação dos fatores mais fortemente relacionados ao fenômeno clima organizacional. É o caso, por exemplo, de Litwin e Stringer (1968), Modelo de Sbragia (1983), Kolb, Rubin e McIntyre (1978), Coda (1997), Luz (2003) e Rizzatti (1995; 2002). Abaixo, são apresentados os principais modelos de análise do clima organizacional e seus respectivos fatores.

- Modelo De Litwin e Stringer (1968): o modelo apresentado por Litwin e Stringer (1968) foi uma das primeiras pesquisas que buscavam identificar os reflexos do clima organizacional e da liderança na satisfação dos funcionários. O modelo é baseado em nove fatores: Estrutura, Responsabilidade, Riscos, Recompensa, CaIor e apoio, Conflito, Desafio, Padrões e Cooperação (LITWIN; STRINGER, 1968; RIZZATTI, 2002; BISPO, 2006; AHRENS, TIMOSSI, DE FRANCISCO, 2014; MARTELO, CLARO, VIEIRA, 2016).

- Modelo De Sbragia (1983): o autor propõe um modelo de pesquisa de clima organizacional composto por vinte fatores, que objetivavam identificar a qualidade do clima organizacional em uma instituição de pesquisa. Os fatores contemplados neste modelo são: Estado de tensão, Conformidade exigida, Ênfase na participação, Proximidade da supervisão, Consideração humana, Adequação da estrutura, Autonomia presente, Recompensas proporcionais, Prestígio obtido, Cooperação existente, Padrões enfatizados, Atitude frente a conflitos, Sentimento de identidade, Tolerância existente, Clareza percebida, Justiça predominante, Condições de progresso, Apoio logístico proporcionado, Reconhecimento proporcionado e Forma de controle (SBRAGIA, 1983; RIZZATTI, 2002; BISPO, 2006; AHRENS; TIMOSSI; DE FRANCISCO, 2014; MARTELO; CLARO; VIEIRA, 2016). 
- Modelo de Kolb, Rubin e Mcintyre (1978): a literatura aponta que o modelo proposto por Kolb, Rubin e McIntyre (1978) é na verdade um aperfeiçoamento do modelo de Litwin e Stringer (1968). O resultado desse aprimoramento é uma escala composta de sete fatores: Conformismo, Clareza organizacional, Calor/Apoio, Liderança, Responsabilidade, Padrões e Recompensas (RIZZATTI, 2002; BISPO, 2006; AHRENS, TIMOSSI, DE FRANCISCO, 2014; MARTELO; CLARO; VIEIRA, 2016).

- Modelo de coda (1997): o modelo apresentado por Coda (1997) resultou de suas pesquisas nas áreas de liderança e motivação. Suas pesquisas envolveram cinco organizações públicas e privadas. Seu modelo contempla dez fatores: Liderança, Compensação, Maturidade empresarial, Colaboração entre áreas funcionais, Valorização profissional, Identificação com a empresa, Processo de comunicação, Sentido do trabalho, Política global de recursos humanos e Acesso (BISPO, 2006; AHRENS; TIMOSSI; DE FRANCISCO, 2014; MARTELO; CLARO; VIEIRA, 2016).

- Modelo de Luz (2003): a proposta de Luz (2003) é apresentar critérios para metodologia de diagnóstico, mensuração e melhoria do clima organizacional. Ou seja, pretendeu-se realizar gestão do clima em dezessete empresas, nacionais e multinacionais, com sede na cidade do Rio de Janeiro. Seu modelo está estruturado em vinte e nove fatores, que buscam identificar variáveis latentes relacionadas ao clima organizacional. Os fatores são: Trabalho realizado, Salário, Benefícios, Integração entre departamentos da empresa, Supervisão, Comunicação, Treinamento, Desenvolvimento, Carreira, Possibilidades de progresso profissional, Relacionamento interpessoal, Estabilidade no emprego, Processo decisório, Condição física de trabalho, Participação, Pagamento de salário, Segurança do trabalho, Objetivos organizacionais, Orientação da empresa para os resultados, Disciplina, Imagem da empresa, Estrutura organizacional, Ética e responsabilidade social, Qualidade e satisfação do cliente, Reconhecimento, Vitalidade organizacional, Direção e estratégias, Valorização dos profissionais, Envolvimento/Comprometimento, Trabalho em equipe, Planejamento organizacional (LUZ, 2003; AHRENS; TIMOSSI; DE FRANCISCO, 2014; MARTELO; CLARO; VIEIRA, 2016).

Os modelos acima apresentados se diferenciam em relação ao tipo de organização em que o clima é avaliado, sendo pública ou privada. No entanto, além desses modelos, Rizzatti (1995) apresenta um que tem como propósito a identificação do clima no ambiente das instituições universitárias.

- Modelo de Rizzatti (1995): A análise do clima organizacional proposta por Rizzatti (1995) contempla variáveis psicossociais que influenciam diretamente na percepção dos funcionários em relação ao construto clima organizacional. O que diferencia o modelo proposto por Rizzatti (1995), quando se pretende analisar o clima em IES, refere-se ao fato de o modelo dele contemplar variáveis presentes nas organizações universitárias que não existem em outras organizações como, por exemplo, objetivos ambíguos e difusos, natureza política, usuários com necessidades individualizadas e diversificadas, tecnologia complexa sensibilidade a fatores dos ambientes externo (RIZZATTI 2002). 


\section{Quadro 1 - Modelo de pesquisa de clima organizacional}

\section{Conceitos e fatores}

CATEGORIA: IMAGEM E AVALIAÇÃO INSTITUCIONAL. CONCEITOS: É tida como "O prestígio de que desfruta a organização junto à comunidade". [...] imagem predominantemente favorável pode ser chamar de prestígio obtido junto à comunidade ou à sociedade (RIZZATTI, 2002, p. 42). FATORES: Satisfação dos usuários, valorização profissional dos empregados, percepção dos objetivos organizacionais, sentimento de identidade, prestígio obtido perante a comunidade e percepção figurativa da organização (RIZZATTI, 2002).

CATEGORIA: DESENVOLVIMENTO DE RECURSOS HUMANOS, BENEFÍCIOS E INCENTIVOS. CONCEITOS: A política de recursos humanos define o modo de atrair, filtrar, manter e avaliar as pessoas que atuam nas organizações. Estas políticas servem de guias ou referências aos componentes de seleção, treinamento, desenvolvimento e avaliação, entre outras práticas que visem ao aprimoramento das pessoas. Políticas de recursos humanos referem-se às maneiras pelas quais a organização pretende lidar com seus membros e, por intermédio deles, atingir os objetivos organizacionais, permitindo condições para o alcance de objetivos organizacionais e individuais. [...] Nas universidades, as políticas de recursos humanas apresentam características complexas e abrangência maior, pois necessitam dedicar atenções especiais ao professor, pesquisador e técnico-administrativos que, nos diversos níveis de responsabilidade, garantem seu funcionamento e permitem o alcance de seus objetivos (RIZZATTI, 2002, p. 45-53). Os planos de serviços e benefícios sociais, geralmente, são planejados para auxiliar o servidor em três áreas de sua vida pessoal e profissional: no exercício do cargo; fora do cargo, mas dentro da empresa; fora da empresa, ou seja, na comunidade. [...] Os benefícios não são apenas onerosos, como nem sempre são o que os empregados desejam. A satisfação com os benefícios tem decaído bastante, porque estes não têm acompanhado as mudanças demográficas da mão de obra. [...] Com relação aos incentivos, são ações ou atitudes que na organização agem como estimulantes do ego do trabalhador, contribuindo para o aumento de sua motivação ou produtividade no trabalho (RIZZATTI, 2002, p. 45-56). FATORES: Condições de progresso funcional, justiça predominante, comprometimento e interesse pelo trabalho, reconhecimento proporcionado e qualidade dos benefícios.

CATEGORIA: ORGANIZAÇÃO E CONDIÇÕES DE TRABALHO. CONCEITOS: Uma ideia básica que contempla o conceito de organização é a coordenação de esforços a serviço da ajuda mútua, uma vez que organização é a coordenação planejada das atividades de uma série de pessoas para a consecução de algum propósito ou objetivo comum, explícito, através da divisão do trabalho em função e através de uma hierarquia de autoridade e responsabilidade. [...] Quanto aos estudos de ambiente ou condições de trabalho, desenvolveram-se em três fases: a primeira fase ocorreu no período de 1940 a 1960, onde os estudos enfatizavam a independência das organizações; num segundo momento, nos anos 1960, predominou a visão da organização como um sistema de lógica fechada; ultimamente, o estudo enfatiza a interdependência cultural e social entre organizações e seus ambientes, onde elas passam a serem vistas como participantes de um grande sistema de relações interorganizacionais (RIZZATTI, 2002, p. 53-68). FATORES: Adequação da estrutura, clareza organizacional, justiça predominante, utilização do tempo, apoio logístico proporcionado, comprometimento e interesse pelo trabalho.

CATEGORIA: RELACIONAMENTO INTERPESSOAL. CONCEITOS: O relacionamento interpessoal refere-se a uma interação ou relação entre duas pessoas ou sistemas de qualquer natureza, de modo que a atividade de cada um está em parte determinada pela atividade do outro (RIZZATTI, 2002, p. 68). FATORES: Consideração humana, cooperação existente e relacionamento existente.

CATEGORIA: SUCESSÃO POLÍTICO-ADMINISTRATIVA E COMPORTAMENTO DAS CHEFIAS. CONCEITOS: Define-se a sucessão político-administrativa como sendo o processo eleitoral para escolha de reitores e outros dirigentes. Quanto ao comportamento das chefias, sabe-se que se trata de uma variável altamente complexa. Desta forma, procurou-se identificar algumas características do chefe e de seu comportamento. A credibilidade e a honestidades são componentes importantes para refletir o comportamento das chefias, mas o conhecimento das atividades também é considerado relevante para refletir o comportamento das chefias e o clima organizacional em universidades. A liderança é necessária em todos os tipos de organização humana, principalmente nas empresas e em cada um de seus departamentos. Ele é igualmente essencial em todas as demais funções de administração: planejamento organizacional, direção e controle. Porém, a liderança é mais relevante na função de direção, àquela que toca mais de perto as pessoas (RIZZATTI, 2002, p. 71-74). FATORES: Consideração humana, ênfase na participação, sucessão administrativa, clareza administrativa, credibilidade, delegação e competência/qualificação.

CATEGORIA: SATISFAÇÃO PESSOAL. CONCEITOS: Levando-se em consideração a relação indivíduo e organização, percebe-se que a satisfação deriva da situação total no trabalho. A satisfação geralmente é usada para expressar resultados já experimentados pelo empregado. [...] a motivação deve ser entendida como um processo amplo, não realizável em curto prazo, e que implica uma postura, uma atitude que deve fluir em todos os níveis da organização, visando a ação para o trabalho provido de sentido e que, consequentemente, gere satisfação dos indivíduos (RIZZATTI, 2002, p. 71-74). FATORES: Jornada de trabalho, sentimento de identidade, prestígio obtido e reconhecimento proporcionado.

Fonte: Elaborado com base em Rizzatti (2002) 


\section{METODOLOGIA}

A pesquisa é classificada quanto aos seus objetivos como descritiva, com abordagem quantitativa na análise dos dados, utilizando-se, quanto aos procedimentos de coleta de dados, de levantamento ou survey. $O$ instrumento de coleta dos dados utilizado foi o desenvolvido por Rizzatti (1995), que está estruturado em 126 perguntas, dividido em duas partes. Na primeira delas, são solicitados os dados sociodemográficos (sexo, faixa etária, nível de escolaridade, tempo de serviço e carga horária semanal). Na segunda parte, são solicitados dados relacionados à percepção do clima organizacional.

O modelo de pesquisa descritivo tem como principal objetivo descrever características de determinada amostra e generalizá-las para população estudada ou para o subgrupo. Ele pode ser do tipo transversal ou longitudinal. No modelo descritivo transversal, a coleta de dados é realizada apenas em um determinado momento, ou seja, faz uma fotografia do fenômeno naquele momento. Já no modelo longitudinal, a coleta de dados é realizada em diversos momentos, que demonstram as características do fenômeno em momento diferentes e como ocorrem ao longo do tempo (BABBIE, 2001; GIL, 2008; MALHOTRA 2011).

A pesquisa do clima organizacional pode se utilizar, basicamente, de quatro métodos ou técnicas para coleta e dados. A primeira delas, utilizada para este trabalho, ocorre por meio de aplicação de questionário ou levantamento, a segunda, entrevista, a terceira, observação direta e a quarta, medições discretas (REGIS FILHO, 2015). As técnicas de coletas de dados "são procedimentos que operacionalizam os métodos. Para todo método de pesquisa, corresponde uma ou mais técnicas e estas estão relacionadas com a coleta de dados, isto é, a parte prática da pesquisa" (RODRIGUES et al., 2014, p. 182).

A determinação da amostragem para esta pesquisa foi feita por amostragem nãoprobabilística. Durante o período de coleta dos dados, compreendido entre os meses de outubro, novembro e dezembro 2018, houve a devolutiva de 309 questionários, com margem de erro de $4,84 \%$, e nível de confiança em $95 \%$. O envio do questionário foi feito aos contatos de e-mails dos funcionários técnico-administrativos da UFAL.

Esta pesquisa utilizou o modelo de análise de clima organizacional proposto por Rizzatti (1995), pois contempla, comparando-o a outros modelos, categorias e fatores específicos para investigação do clima em ambientes universitários. O instrumento é estruturado em seis categorias que se subdivide em 31 fatores. As categorias contempladas no referido modelo são Imagem e avaliação institucional; Desenvolvimento de recursos humanos, benefícios e incentivos; Organização e condições de trabalho; Relacionamento interpessoal; Sucessão político-administrativa; e Satisfação pessoal. O questionário utiliza a escala do tipo Liberta (1932), de 5 pontos, em que o continiuum de escalonamento das respostas foi distribuído de 1 a 5, em que o número 1 (um) representa o valor mínimo, que corresponde a nada, ruim ou nunca, e o número 5 (cinco) representa o valor máximo e corresponde a muitíssimo, ótimo ou sempre.

Optou-se pela abordagem quantitativa, já que tal propósito é avaliar as conexões estabelecidas entre variáveis, de modo a comprovar a relação existente de dependência e/ou independência de tais variáveis (FLICK, 2013). Já Rodrigues et al. (2014, p. 56) destacam que essa abordagem "[...] está relacionada à quantificação de dados obtidos mediante pesquisa. Utiliza-se na pesquisa de recursos e técnicas estatísticas.". Por sua vez, Sampieri, Collado e Lucio (2013) mencionam que a abordagem quantitativa busca descrever, explicar e prevê fenômenos. 
Segundo Vieira (2011, p. 4) “[...] população ou universo é o conjunto de unidades sobre o qual desejamos obter informação. Amostra é todo subconjunto de unidades retiradas de uma população para obter a informação desejada.". Seguindo este raciocínio, tem-se que, neste estudo, definiu-se a população como sendo os funcionários técnicoadministrativos da UFAL, ocupantes dos cargos de níveis fundamental, médio e superior efetivos, regidos pela Lei no 8.112/90. Lotados nos Campi da universidade, excetuando-se apenas aqueles lotados no Hospital Universitário Prof. Alberto Antunes (HUPPA). Também foram excluídos os funcionários regidos por regime celetista ou contrato temporário. Dessa forma, a população foi composta por 1.246 funcionários.

Os dados desta pesquisa foram analisados por meio da utilização de técnicas estatísticas multivariadas, em que se combinam técnicas estatísticas ao mesmo tempo para analisar múltiplas variáveis relacionadas à população estudada (HAIR et al., 2009).

Para tabulação e análise dos dados, foram utilizados os aplicativos Microsoft Office Excel e Statistical Package for Social Science versão 21 (SPSS). Com isso, primeiro foi realizada a análise descritiva sociodemográfica da amostra. Em seguida, foi realizada a análise do índice, ou seja, o score extraído de cada categoria e do construto clima organizacional. Por fim, foi realizada análise fatorial, que buscou identificar a relação entre as categorias e seus respectivos fatores, de modo a verificar a relação entre tais variáveis, ou seja, identificar se os fatores apresentam correlações com suas respectivas categorias (HAIR et al., 2009).

O Quadro 2 apresenta os parâmetros utilizados para verificação da normalidade dos dados, permitindo-se sua submissão ao processo de fatoração.

Quadro 2 - Procedimentos de verificação de adequabilidade dos dados, 2018

\begin{tabular}{|l|l|}
\hline \multicolumn{2}{|c|}{ Adequação do modelo } \\
\hline Kaiser-Meyer-Olkin (KMO) & Valores entre 0 e 1 \\
\hline Teste de esfericidade de Bartlett & $\begin{array}{l}\text { quanto mais próximo de zero }(0,0) \text {, maior da adequa- } \\
\text { ção da análise fatorial }\end{array}$ \\
\hline $\begin{array}{l}\text { Matriz anti-imagem - medida de adequação da } \\
\text { amostra (MAA) }\end{array}$ & $\begin{array}{l}\text { quanto mais próximo de } 1 \text { o valor da MMA, mais ade- } \\
\text { quada é a análise fatorial }\end{array}$ \\
\hline Matriz de comunalidade & $\begin{array}{l}\text { MSA < } 0,5 \text { não é considerado } \\
\text { MSA } \geq 0,8 \text { é desejável }\end{array}$ \\
\hline
\end{tabular}

Fonte: Dados da pesquisa (2018).

\section{RESULTADOS}

Esta seção foi dedicada à análise dos dados, utilizando-se de técnicas multivariadas (análise descritiva e fatorial). Inicialmente, fez-se a apresentação descritiva dos dados sociodemográficos da amostra, composta por 309 respondentes, que representou $24.79 \%$ da totalidade da população, em seguida, foi feita a análise descritiva dos índices das categorias e do construto clima organizacional, seguida da análise fatorial com intuito de se identificar o agrupamento e correlações entre os fatores e categorias presentes no modelo de Rizzatti (1995).

Quanto ao sexo, 41\% (128) são do sexo masculino e 59\% (181) correspondem ao sexo feminino. Vale destacar que a proporção de pessoas do sexo masculino em relação ao sexo feminino foi a mesma identifica na população, $59 \%$ (1.038) correspondem ao sexo feminino e $41 \%$ (731) do sexo masculino. 
No que se refere à faixa etária, os dados revelaram que a amostra é composta por $53 \%$ de funcionários com mais de 36 anos e $47 \%$ de pessoas que têm entre 18 e 36 anos.

Observou-se que $30 \%$ dos funcionários da amostra têm até cinco anos de serviço; $38 \%$, de cinco a 10 anos; $16 \%$, de 10 a 20 anos e $16 \%$, mais de 20 anos de serviços prestados. Constatou-se, dessa forma, que a maioria dos funcionários da amostra são pessoas relativamente com pouco tempo de serviço, considerando as exigências mínimas para aposentadoria (a partir dos 30 ou 35 anos).

Já no que se refere ao nível de escolaridade, constatou-se que $54 \%$ dos respondentes possuem pós-graduação lato sensu, acompanhados de $19 \%$ de funcionários com pósgraduação stricto sensu (mestrado) e $18 \%$ possuem nível superior. Os detentores de pósgraduação stricto sensu (doutorado) representam $4 \%$ da amostra e $5 \%$ têm ensino médio. Esses percentuais se redistribuem em relação à totalidade da população, pois, conforme o RG/Ufal/2017, a maioria dos técnicos tem nível superior (34\%).

Em relação à carga horária, os dados coletados demonstraram que $54 \%$ prestam 40 horas semanais, enquanto $46 \%$ perfazem 30 horas por semana. Essa diferenciação que se dá entre a carga horária dos funcionários do segmento administrativo ocorre pela flexibilização permitida na Resolução no 53/2017 - Consuni/Ufal, de 09 de outubro de 2017, que institui a jornada de trabalho de seis horas diárias e 30 horas semanais para o segmento técnicoadministrativo da UFAL.

\subsection{Análise fatorial}

\section{- Primeira fatorial com 31 variáveis}

A primeira tentativa envolveu todas as 31 variáveis do modelo (F1CAT1 - Sentimento de identidade, F2CAT1 - Satisfação do usuário, F3CAT1 - Prestígio obtido, F4CAT1 - Percepção figurativa, F5CAT1 - Percepção dos objetivos, F1CAT2 - Condições de progresso funcional, F2CAT2 - Reconhecimento proporcionado, F3CAT2 - Justiça predominante, F4CAT2 Comprometimento e interesse pelo trabalho, F5CAT2 - Qualidade dos benéficos, F1CAT3 Adequação da estrutura, F2CAT3 - Clareza organizacional, F3CAT3 - Apoio logístico proporcionado, F4CAT3 - Terceirização, F5CAT3 - Justiça predominante, F6CAT3 - Comprometimento e interesse, F7CAT3 - Utilização do tempo, F1CAT4 - Relacionamento existente, F2CAT4 Cooperação existente, F3CAT4 - Consideração humana, F1CAT5 - Sucessão políticoadministrativa, F2CAT5 - Credibilidade, F3CAT5 - Competência e qualificação, F4CAT5 - Delegação, F5CAT5 - Clareza percebida, F6CAT5 - Ênfase na participação, F7CAT5 - Consideração humana, F1CAT6 - Satisfação no trabalho, F2CAT6 - Jornada de trabalho, F3CAT6 - Prestígio obtido, F4CAT6 - Reconhecimento proporcionado).

Essa primeira tentativa buscou verificar quais seriam as dimensões latentes ou construtos que melhor explicassem as correlações entre todas as variáveis. Para tanto, foram realizados os testes medida de adequação da amostra de Kaiser-Meyer-Olhinho (KMO) e Bartlett's test of sphericity (teste de esfericidade de Bartlett).

De acordo com os testes realizados, o KMO foi 0,927. Já o teste de esfericidade de Bartlett (Sig.) apresentou resultado 0,0. Na matriz Anti-image correlation (correlação de antiimagem), verificou-se que todas as variáveis possuíam KMO alto e a análise da matriz de Anti-image Correlation não indicou a exclusão de variáveis. 
No entanto, ao se analisar a comunalidade, que verifica a proporção de variância comum nas variáveis excelentes (FIELD, 2009; HAIR et al., 2009; MALHOTRA, 2011), identificouse que as variáveis F3CAT2 - Justiça predominante $(0,461)$, F5CAT2 - Qualidade dos benéficos $(0,462)$, F2CAT3 - Clareza organizacional $(0,431)$, F4CAT3 - Terceirização, F5CAT3 - Justiça predominante, F7CAT3 - Utilização do tempo, F1CAT5 - Sucessão político-administrativa, e F2CAT6 - Jornada de trabalho, apresentaram carga fatorial baixas, abaixo de 0,5 . Os valores abaixo de 0,50 são tidos como muito pequenos, apontando para a necessidade de retiradas dessas variáveis da análise de repetir o processo (BEZERRA; CORRAR; 2006).

Com a variância total explicada foi possível identificar que há explicação de $62,50 \%$ do modelo. Diante da exclusão de algumas variáveis, conforme demonstrado anteriormente, houve a necessidade de se repetir a análise fatorial com o modelo contendo 23 variáveis.

\section{- Segunda fatorial com 23 variáveis}

Mesmo com um alto grau de explicação do modelo pelas variáveis $(62,50 \%)$ verificado na matriz de variância total explicada, decidiu-se excluir aquelas com valores abaixo de 0,50, por serem considerados muito pequenos e seguir para próxima tentativa utilizando-se as demais 23 variáveis. Com isso, repetiram-se os testes de normalidade dos dados.

$\mathrm{O}$ teste $\mathrm{KMO}$, apresentou valores importantes $(0,932)$ e o Teste de esfericidade de Bartlett $(0,0)$ também foi significativo, pois é menor que 0 . Tendo os pré-requisitos respeitados, seguiu-se com a análise.

Verificou-se que, após a exclusão de 8 variáveis, o KMO obteve valor superior ao modelo anterior (0,932). A próxima análise foi feita na matriz de Anti-image correlation, (correlação de anti-imagem). Os resultados apontaram que o KMO individual de todas as variáveis continua altos, próximos de 1.

Considerando que as variáveis apresentaram valores significativos, observou-se na matriz de comunalidade, que uma variável (F4CAT1 - Percepção figurativa) apresentou valor baixo $(0,473)$, abaixo de 0,50 . A matriz de variância total explicada possibilitou verificar que o modelo é explicado em $69,66 \%$, percentual considerado expressivo. Dessa forma, tendo em vista que a matriz de comunalidades trouxe a indicação de exclusão de uma variável, houve a necessidade de se repetir a análise fatorial.

\section{- Terceira fatorial com 22 variáveis}

Mesmo obtendo-se alto valor explicativo do modelo com 23 variáveis, tomou-se a decisão de retirar a variável F4CAT1 - Percepção figurativa, do processo e repetir a análise novamente, agora com 22 variáveis. Foi repetido o teste KOM e Teste de esfericidade de Bartlett, Tabela 1, que apresentaram resultados de normalidade da amostra, com KMO 0,930 e Sig. 0,00.

Tabela 1 - Teste de KMO e Bartlett, análise fatorial

\begin{tabular}{lcc}
\hline \multicolumn{2}{c}{ Medida Kaiser-Meyer-Olkin de adequação de amostragem. } &, 930 \\
Teste de esfericidade de Bartlett & Qui-quadrado aprox. & 4641,495 \\
\hline
\end{tabular}


A matriz de anti-imagem, Tabela 2, continuou a demonstrar elevado KMO individual das variáveis, todos próximos de 1.

Tabela 2 - Anti-image correlation (correlação de anti-imagem), análise fatorial

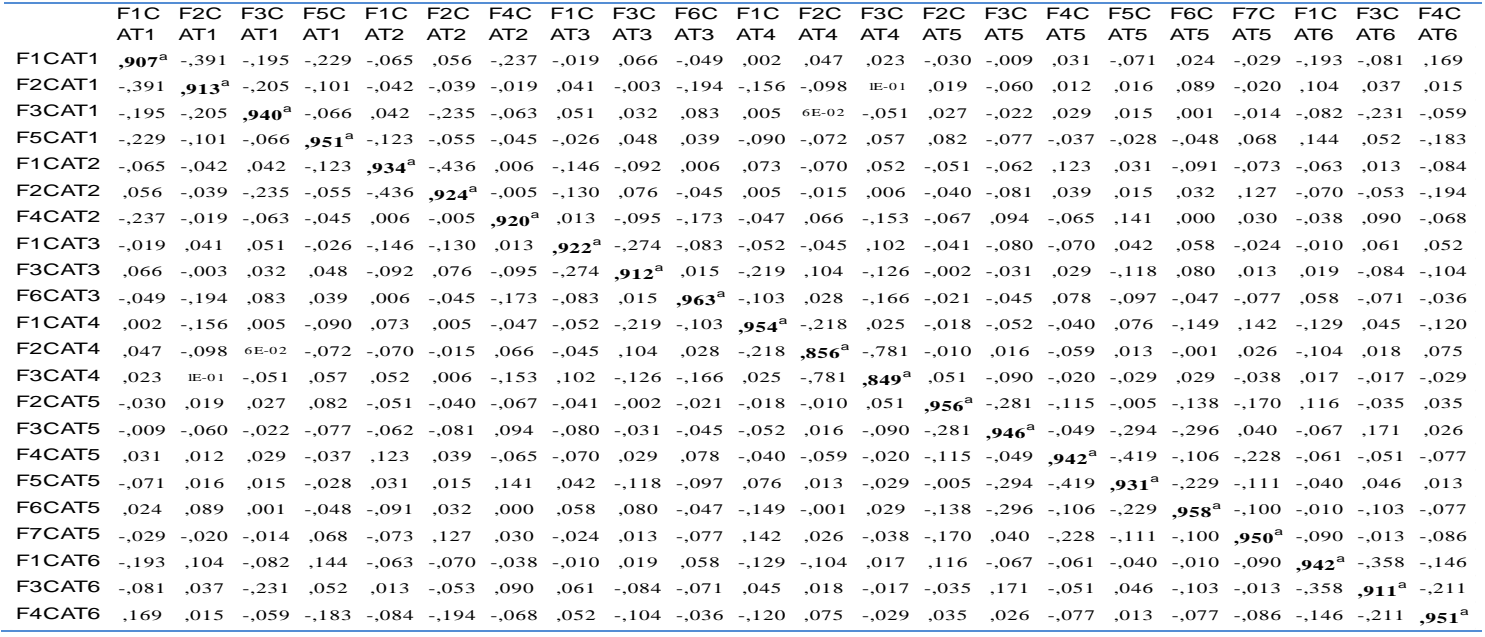

A Tabela 3 demonstra os valores encontrados relacionados à comunalidade após a exclusão do fator F4CAT1 - Percepção figurativa. Os números apontam para o modelo adequado, com valores superiores a 0.50 em todas as variáveis.

Tabela 3 - Matriz de comunalidades, análise fatorial

\begin{tabular}{|llc|}
\hline \multicolumn{1}{c}{ Fator } & Inicial & Extração \\
\hline F1CAT1 - Sentimento de identidade & 1,000 &, 763 \\
\hline F2CAT1 - Satisfação do usuário & 1,000 &, 737 \\
\hline F3CAT1 - Prestígio obtido & 1,000 &, 709 \\
\hline F5CAT1 - Percepção dos objetivos & 1,000 &, 590 \\
\hline F1CAT2 - Condições de progresso funcional & 1,000 &, 678 \\
\hline F2CAT2 - Reconhecimento proporcionado & 1,000 &, 703 \\
\hline F4CAT2 - Comprometimento e interesse pelo trabalho & 1,000 &, 513 \\
\hline F1CAT3 - Adequação da estrutura & 1,000 &, 708 \\
\hline F3CAT3 - Apoio logístico proporcionado & 1,000 &, 594 \\
\hline F6CAT3 - Comprometimento e interesse & 1,000 &, 585 \\
\hline F1CAT4 - Relacionamento existente & 1,000 &, 666 \\
\hline F2CAT4 - Cooperação existente & 1,000 &, 825 \\
\hline F3CAT4 - Consideração humana & 1,000 &, 861 \\
\hline F2CAT5 - Credibilidade & 1,000 &, 675 \\
\hline F3CAT5 - Competência e qualificação & 1,000 &, 818 \\
\hline F4CAT5 - Delegação & 1,000 &, 791 \\
\hline F5CAT5 - Clareza percebida & 1,000 &, 827 \\
\hline F6CAT5 - Ênfase na participação & 1,000 &, 798 \\
\hline F7CAT5 - Consideração humana & 1,000 &, 641 \\
\hline F1CAT6 - Satisfação no trabalho & 1,000 &, 745 \\
\hline F3CAT6 - Prestígio obtido & 1,000 &, 775 \\
\hline F4CAT6 - Reconhecimento proporcionado & 1,000 &, 688 \\
\hline
\end{tabular}

Fonte: Dados da pesquisa (2018). Método de extração: análise do componente principal. 
Já na matriz de variância total explicada (Tabela 4), verificou-se que o modelo consegue explicar $71,32 \%$ da variância dos dados originais.

Tabela 4 - Variância total explicada, análise fatorial

\begin{tabular}{|c|c|c|c|c|c|c|c|c|c|}
\hline \multirow[t]{2}{*}{ 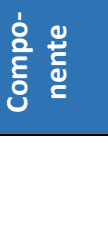 } & \multicolumn{3}{|c|}{ Valores próprios iniciais } & \multicolumn{3}{|c|}{$\begin{array}{c}\text { Somas de extração de } \\
\text { carregamentos ao } \\
\text { quadrado }\end{array}$} & \multicolumn{3}{|c|}{$\begin{array}{l}\text { Somas rotativas de } \\
\text { carregamentos ao quadrado }\end{array}$} \\
\hline & Total & $\begin{array}{l}\text { \% de } \\
\text { variân- } \\
\text { cia }\end{array}$ & $\begin{array}{c}\% \text { cumula- } \\
\text { tiva }\end{array}$ & $\begin{array}{l}\text { To- } \\
\text { tal }\end{array}$ & $\begin{array}{l}\% \text { de } \\
\text { vari- } \\
\text { ância }\end{array}$ & $\begin{array}{c}\% \text { cumula- } \\
\text { tiva }\end{array}$ & $\begin{array}{l}\text { To- } \\
\text { tal }\end{array}$ & $\begin{array}{l}\text { \% de } \\
\text { variân- } \\
\text { cia }\end{array}$ & $\begin{array}{c}\% \text { cumula- } \\
\text { tiva }\end{array}$ \\
\hline 1 & 9,934 & 45,15 & 45,15 & 9,93 & 45,15 & 45,15 & 4,69 & 21,34 & 21,34 \\
\hline 2 & 2,235 & 10,15 & 55,31 & 2,23 & 10,15 & 55,31 & 3,48 & 15,85 & 37,20 \\
\hline 3 & 1,297 & 5,895 & 61,209 & 1,29 & 5,895 & 61,20 & 2,81 & 12,77 & 49,98 \\
\hline 4 & 1,140 & 5,184 & 66,393 & 1,14 & 5,184 & 66,39 & 2,63 & 11,95 & 61,93 \\
\hline 5 & 1,084 & 4,928 & 71,320 & 1,08 & 4,928 & 71,32 & 2,06 & 9,38 & 71,32 \\
\hline 6 & ,785 & 3,569 & 74,889 & & & & & & \\
\hline 7 & ,624 & 2,834 & 77,723 & & & & & & \\
\hline 8 & ,591 & 2,687 & 80,411 & & & & & & \\
\hline 9 & ,512 & 2,325 & 82,736 & & & & & & \\
\hline 10 & ,483 & 2,195 & 84,931 & & & & & & \\
\hline 11 & ,463 & 2,106 & 87,037 & & & & & & \\
\hline 12 & ,390 & 1,775 & 88,812 & & & & & & \\
\hline 13 & ,382 & 1,739 & 90,550 & & & & & & \\
\hline 14 & 371 & 1,688 & 92,238 & & & & & & \\
\hline 15 & ,302 & 1,373 & 93,611 & & & & & & \\
\hline 16 & 275 & 1,252 & 94,863 & & & & & & \\
\hline 17 & ,254 & 1,155 & 96,018 & & & & & & \\
\hline 18 & 231 & 1,052 & 97,070 & & & & & & \\
\hline 19 & 220 & 1,000 & 98,070 & & & & & & \\
\hline 20 & 170, & 772 & 98,842 & & & & & & \\
\hline 21 & 147, & 670 & 99,512 & & & & & & \\
\hline 22 & 107 & ,488 & 100,000 & & & & & & \\
\hline
\end{tabular}

Fonte: Dados da pesquisa (2018). Método de extração: análise do componente principal.

Observou-se que houve um aumento significativo do percentual de variância total do modelo com 22 variáveis $(71,23 \%)$, em relação aos modelos anteriores, em que o primeiro apresentou $62,50 \%$ e o segundo, $69,66 \%$.

A matriz de componente rotativa (Tabela 5) traz a classificação e organização das variáveis, distribuídas pelas respectivas dimensões latentes. Pelo modelo conseguido, é possível verificar readequação das variáveis e sua carga fatorial. O novo modelo se estruturou em 22 fatores com 5 dimensões, diferentemente do modelo original em que haviam 31 fatores e 6 dimensões. Esses fatores se organizam da seguinte forma: Dimensão 1: F5CAT5; F4CAT5; F6CAT5; F3CAT5; F2CAT5; F7CAT5; Dimensão 2: F2CAT1, F1CAT1, F5CAT1, F3CAT1, F4CAT2; Dimensão 3: F3CAT6; F1CAT6; F4CAT6, F2CAT2; Dimensão 4: F3CAT4; F2CAT4; F1CAT4; F6CAT3; e, Dimensão 4: F1CAT3; F3CAT3; F1CAT2. Após a reorganização das variáveis no melhor modelo, foi possível perceber que algumas variáveis se reorganizaram nas dimensões.

Após os testes realizados para se alcançar o modelo que melhor se adequasse ao propósito de mensurar o fenômeno, a relação estabelecida no modelo inicial se manteve no que se refere à categoria imagem e avaliação institucional. Os fatores demonstraram alta carga fatorial e permaneceram alinhados à mesma categoria, da seguinte forma: Satisfação do usuário 0,821; Sentimento de identidade 0,805; Prestígio obtido 0,603; Percepção dos objetivos 0,651; Percepção figurativa 0,572. 
Tabela 5 - Matriz de componente rotativa ${ }^{a}$, análise fatorial

\begin{tabular}{|c|c|c|c|c|c|}
\hline \multirow[t]{2}{*}{ Fator } & \multicolumn{5}{|c|}{ Componente } \\
\hline & 1 & 2 & 3 & 4 & 5 \\
\hline F5CAT5 & ,845 & & & & \\
\hline F4CAT5 & ,814 & & & & \\
\hline F6CAT5 & ,789 & & & & \\
\hline F3CAT5 & 771 & & & & \\
\hline F2CAT5 & ,767 & & & & \\
\hline F7CAT5 & ,761 & & & & \\
\hline F2CAT1 & & ,821 & & & \\
\hline F1CAT1 & & ,805 & & & \\
\hline F5CAT1 & & ,651 & & & \\
\hline F3CAT1 & & ,603 & ,574 & & \\
\hline F4CAT2 & & ,572 & & ,412 & \\
\hline F3CAT6 & & & ,833 & & \\
\hline F1CAT6 & & & ,695 & & \\
\hline F4CAT6 & & & 644 & & \\
\hline F2CAT2 & & ,445 & ,518 & & ,456 \\
\hline F3CAT4 & & & & ,843 & \\
\hline F2CAT4 & & & & ,810 & \\
\hline F1CAT4 & & & & ,550 & \\
\hline F6CAT3 & & ,440 & & ,460 & \\
\hline F1CAT3 & & & & & ,800 \\
\hline F3СAT3 & & & & & ,636 \\
\hline F1CAT2 & & ,418 & & &, 532 \\
\hline
\end{tabular}

Fonte: Dados da pesquisa (2018). Método de extração: Análise do Componente principal. Método de rotação: Varimax com normalização de Kaiser. ${ }^{a}$. Rotação convergida em 7 iterações.

Já na categoria desenvolvimento de recursos humanos, benefícios e incentivos, os resultados da análise fatorial demonstraram expressiva baixa carga fatorial para quatro fatores. Essa categoria era, inicialmente, composta por cinco fatores. Apenas o fator reconhecimento proporcionado permaneceu no modelo, no entanto, indicou para forte adequação à categoria satisfação pessoal. Dessa forma, a categoria desenvolvimento de recursos humanos, benefícios e incentivos não se manteve ao novo modelo, indicando-se para sua exclusão.

A categoria organização e condições de trabalho no modelo inicial proposto era apresentada por sete fatores, após as tentativas de readequação do modelo, apenas três fatores - comprometimento e interesse $(0,460)$ adequação da estrutura $(0,800)$ e apoio logístico proporcionado $(0,636)$ - permaneceram, pois apresentaram carga fatorial relevante. No entanto, os resultados apontaram para a necessidade de reorganização desses fatores dentro das categorias, sugerindo que o fator comprometimento e interesse se adequasse melhor com a categoria relacionamento interpessoal, enquanto os fatores adequação da estrutura e apoio logístico proporcionado permanecessem adequados à categoria organização e condições de trabalho.

A categoria relacionamento interpessoal se relacionava apenas com três fatores. Posteriormente as tentativas das análises, os resultados indicaram a permanência desses fatores e o fator comprometimento e interesse também se agregou a esta categoria. Suas cargas fatoriais apresentaram resultados importantes (comprometimento e interesse 0,460; relacionamento existente 0,550 ; cooperação existente 0,810 ; e, consideração humana 0,843).

Inicialmente composta por sete fatores, a categoria sucessão político-administrativa, após análise das cargas fatoriais e readequação do modelo, indicou para a permanência de 
seis fatores - credibilidade $(0,767)$, competência e qualificação $(0,771)$, delegação $(0,814)$, clareza percebida $(0,845)$, ênfase na participação $(0,789)$ e consideração humana $(0,761)$. Apontando para a exclusão do fator denominado sucessão político-administrativa.

\subsection{Análise descritiva dos índices das categorias do clima organizacional - medida de ten- dência central: média}

Para se chegar ao índice de cada fator, fez-se a média aritmética, ou seja, tendo-se um conjunto de $n$ valores $x 1, \times 2, \times 3, \ldots, x n$ de uma variável $x$, é o quociente entre a soma desses valores e no número deles (POCINHO, 2014). Assim, inicialmente, realizou-se o procedimento de separação das questões com seus respectivos fatores, em seguida fez-se a média aritmética de cada fator, chegando-se aos índices apresentados no gráfico abaixo.

Gráfico 1 - Índices dos fatores da pesquisa de clima organizacional da UFAL

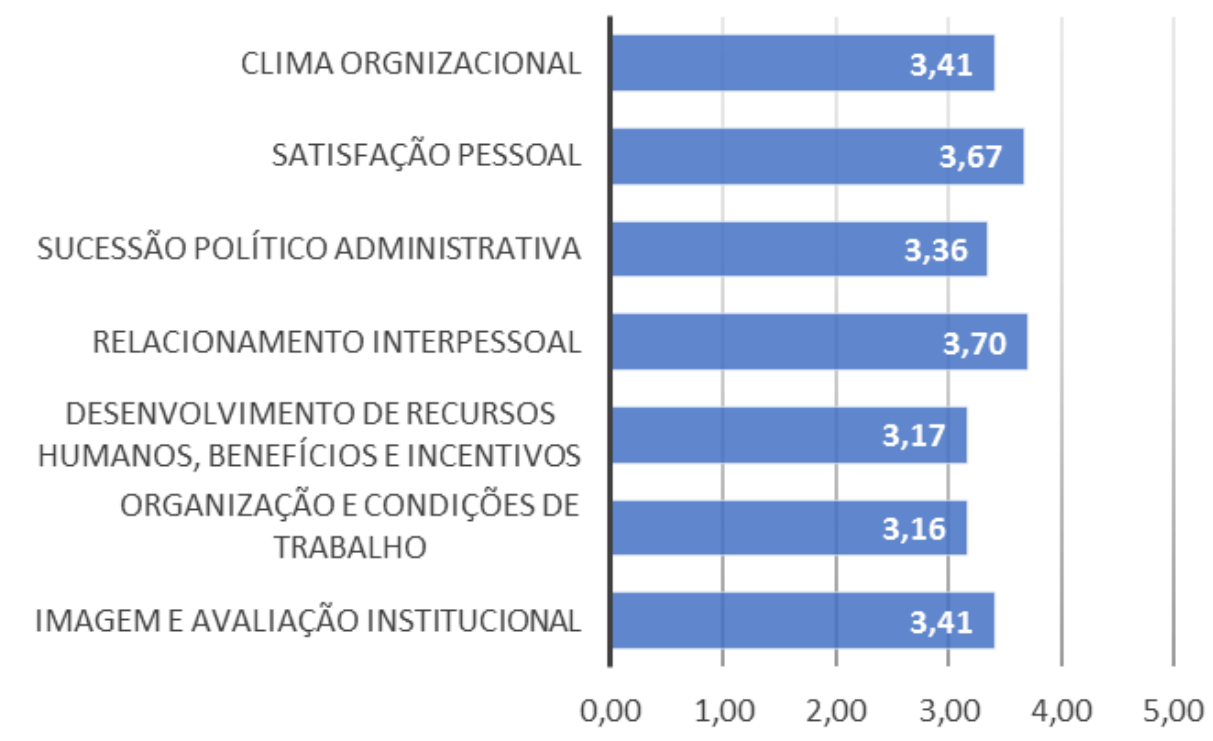

Fonte: Dados da pesquisa (2018).

A categoria imagem e avaliação institucional, que buscou identificar os dados e informações sobre o prestígio de que desfruta a organização, tanto internamente quanto externamente, a partir da percepção dos funcionários, e envolveu os fatores sentimento de identidade, satisfação do usuário, prestígio obtido, percepção figurativa e percepção dos objetivos (RIZZATTI, 2002), obteve avaliação medianamente positiva $(3,41)$, apontando para avaliação positiva da imagem da universidade e atribuindo reflexos positivos sobre o clima organizacional.

A categoria desenvolvimento de recursos humanos, benefícios e incentivos diz respeito ao conjunto de princípios, estratégias e técnicas utilizadas, em especial, pela Gestão de Recursos Humanos para manter, capacitar e treinar os funcionários, obteve avaliação medianamente positiva $(3,17)$. Dessa forma, percebe-se que a avaliação feita pelos funcionários no que se referem às políticas institucionais são, de forma relativa, satisfatórias. Essa categoria é composta pelos fatores condições de progresso funcional, reconhecimento proporcionado, justiça predominante, comprometimento pelo trabalho e qualidade dos benefícios. 
Por sua vez, a categoria organização e condições de trabalho $(3,16)$ obteve média com tendência à avaliação positiva pelos funcionários. Nesta categoria, são avaliados os fatores adequação da estrutura, clareza organizacional, apoio logístico proporcionado, terceirização, justiça predominante, comprometimento e interesse e utilização do tempo.

A categoria relacionamento interpessoal $(3,70)$ alcançou média positiva, demonstrando a existência de relacionamento medianamente positivo. Esta categoria verifica o relacionamento existente entre as pessoas, individual e coletivamente e é composta pelos fatores relacionamento existente, cooperação existente e consideração humana.

Já a categoria sucessão político-administrativa $(3,36)$ teve índice relativamente satisfatório. Nessa categoria, busca-se verificar como se dão os mecanismos de ocupação dos cargos dentro da universidade, englobando designações, indicação ou eleição. Ela envolve os fatores sucessão político-administrativa, credibilidade, competência e qualificação, delegação, clareza organizacional, ênfase na participação e consideração humana.

A categoria satisfação pessoal $(3,67)$ obteve índice consideravelmente positivo. Neste item, busca-se identificar o grau de contentamento dos funcionários em relação ao reconhecimento percebido pela organização sobre as funções e atribuições relacionadas ao cargo. Esta categoria envolve os fatores satisfação no trabalho, jornada de trabalho, prestígio obtido e reconhecimento proporcionado.

\subsection{Medida de tendência central - moda - análise descritiva da distribuição dos índices das categorias do clima organizacional}

Por fim, o construto clima organizacional recebeu avaliação expressivamente concentrada entre os índices 3,00 e 3,99, com 74,8\% dos respondentes. O clima organizacional foi tido como positivo, pois, de acordo com os dados coletados, a média obtida foi consideravelmente tendente ao clima bom ou satisfatório $(3,41)$.

Tabela 6 - Distribuição dos índices de classificação do clima

\begin{tabular}{ccc} 
Classificação & Frequência & Porcentual \\
\hline 1,00 a 1,99 & 3 & 1,0 \\
2,00 a 2,99 & 48 & 15,5 \\
3,00 a 3,99 & 231 & 74,8 \\
4,00 a 5,00 & 27 & 8,7 \\
\hline Total & 309 & 100,0 \\
\hline
\end{tabular}

Fonte: Dados da pesquisa (2018).

Os reflexos das avaliações medianamente positivas realizadas pelos respondentes em relação às categorias do clima organizacional em que imagem e avaliação institucional $(3,41)$, desenvolvimento de recursos humanos, benefícios e incentivos $(3,17)$, organização e condições de trabalho $(3,16)$, relacionamento interpessoal $(3,7)$, sucessão políticoadministrativa e comportamento das chefias $(3,36)$, e satisfação pessoal $(3,67)$ alcançaram índices superiores à média, repercutiu positivamente no clima organizacional da UFAL. 


\section{CONSIDERAÇÕES FINAIS}

A pesquisa se propôs analisar o clima organizacional da UFAL, a partir da percepção dos funcionários técnico-administrativos, utilizando-se de métodos estatísticos e análises multivariadas.

Os dados permitiram a identificação do índice do clima organizacional medianamente positivo $(3,41)$ da UFAL, bem como das categorias que apresentam importantes reflexos sobre o clima positivo: imagem e avaliação institucional $(3,41)$, desenvolvimento de recursos humanos, benefícios e incentivos $(3,17)$, organização e condições de trabalho $(3,16)$, relacionamento interpessoal $(3,70)$, sucessão político-administrativa $(3,36)$ e satisfação pessoal $(3,67)$, a partir da percepção dos seus funcionários técnico-administrativos. Predominantemente, os fatores demonstraram tendência de avaliação medianamente positiva dessas categorias.

Chega-se, portanto, à conclusão de que a avaliação dos fatores e categorias que possuem importantes reflexos sobre o construto clima organizacional é predominantemente positiva. Ou seja, os funcionários técnico-administrativos da UFAL que responderam ao questionário da pesquisa avaliam positivamente os componentes que refletem na boa percepção do ambiente de trabalho. Importante mencionar que a distribuição do quantitativo de avaliações expressivamente positivas do clima organizacional se deu entre os índices 3,00 e 3,99, em que $74,8 \%$ dos respondentes fizeram avaliação de que percebem o clima bom.

Em contrapartida, a concentração de avaliações negativas do clima foi pequena, pois $16,5 \%$ dos respondentes percebem o clima com índices inferiores à média $(3,00)$. E, desses $16,5 \%$, tão somente $1 \%$ avaliaram o clima com notas entre 1,00 e 1,99.

Outra técnica que se demonstrou adequada para análise dos dados foi a fatorial. Diversos testes foram realizados e para verificar a normalidade dos dados. Foram realizadas três tentativas de análise fatorial para se alcançar a melhor readequação dos fatores e apresentação do modelo mais apropriado. Na terceira tentativa, houve significativa adequação dos fatores. De início, foram realizados os testes de normalidade dos dados. Com 22 variáveis, o teste $\mathrm{KMO}$ e Teste de esfericidade de Bartlett apresentaram resultados de normalidade da amostra, com KMO 0,930 e Sig. 0,00. A matriz de anti-imagem continuou a demonstrar elevado KMO individual das variáveis, todos próximos de 1. A matriz de comunalidade resultou em valores que apontaram para o modelo adequado, com valores superiores a 0.50 em todas as variáveis. Já na matriz de variância total explicada, verificou-se que o novo modelo consegue explicar $71,32 \%$ da variância dos dados originais.

Já a matriz de componente rotativa trouxe a classificação e organização das variáveis, distribuídas pelas respectivas dimensões latentes. Pelo modelo conseguido, é possível verificar readequação das variáveis e sua carga fatorial. O novo modelo se estruturou em 22 fatores. Como houve uma análise fatorial, verificou-se a necessidade de readequação das variáveis no modelo e a exclusão de algumas variáveis. A relação estabelecida no modelo inicial se manteve no que se refere à categoria imagem e avaliação institucional. Os fatores demonstraram alta carga fatorial e permaneceram alinhados à mesma categoria, da seguinte forma: Satisfação do usuário 0,821; sentimento de identidade 0,805 ; prestígio obtido 0,603; percepção dos objetivos 0,651; percepção figurativa 0,572.

Por outro lado, no que se refere à categoria desenvolvimento de recursos humanos, benefícios e incentivos, os resultados da análise fatorial demonstraram expressiva baixa carga fatorial para quatro fatores. Essa categoria era, inicialmente, composta por cinco fatores. Apenas o fator reconhecimento proporcionado permaneceu no modelo, no entanto, indicou 
para forte adequação à categoria satisfação pessoal. Dessa forma, a categoria desenvolvimento de recursos humanos, benefícios e incentivos não se manteve ao modelo original, indicando-se para sua exclusão do instrumento.

As demais categorias, relacionamento interpessoal, sucessão político-administrativa, organização e condições de trabalho e satisfação pessoal, apresentaram readequação das variáveis, no entanto, permaneceram no modelo. Dessa forma, a análise fatorial dos 31 fatores propostos no modelo teórico inicial indica para a readequação do modelo, reduzindo-o a 22 fatores, com expressivas cargas fatoriais.

Diante do exposto, considera-se importante a tomada de ações institucional pela gestão de recursos humanos da UFAL no sentido de apresentar melhores condições de trabalho para seus funcionários para aquelas variáveis tidas como prejudicadas. A abordagem quantitativa dos dados definida na metodologia foi uma limitação da pesquisa, pois restringiu as possibilidades de se identificar com maior profundidade e subjetividade como ocorrem os fenômenos estudados, em especial, o clima organizacional. Considerando-se que, para este trabalho, a amostragem escolhida foi a não-probabilística, não é possível generalizar os resultados, apresentando-se também como uma das limitações. Soma-se a isto a população escolhida, neste trabalho, limitando-se aos funcionários técnico-administrativos, não envolvendo os professores e os discentes.

Sugere-se, diante do exposto, que outras pesquisas sejam realizadas e contemplem as categorias dos docentes e discentes para que se chegue ao mais próximo da real percepção do clima organizacional no âmbito da UFAL. Também se sugere atribuir novas denominações às categorias do novo modelo resultante da análise fatorial, com cinco categorias e excluir variáveis com mesma denominação ou renomeá-las. Em outra frente, sugere-se a realização de outras pesquisas que se utilize o modelo reduzido neste trabalho para que se verifique a consistência dos resultados, o grau de explicação dos fenômenos e sua adequabilidade.

\section{REFERÊNCIAS}

AHRENS, R. B.; TIMOSSI, L. da S.; DE FRANCISCO, A. C. Analise comparativa entre modelos de pesquisa em clima organizacional: comparative analysis between models of research in organizational climate. Espacios, v. 35, n. 9, 2014.

ARABAC, I. B. Academic and administration personnel's perceptions of organizational climate: Sample of Educational Faculty of Firat University. Procedia Social and Behavioral Sciences, 2, p. 4445-4450, 2010.

BABBIE, E. Métodos de Pesquisas em Survey. Belo Horizonte: Editora UFMG, 2001.

BARROS, P. L.; MELO, J. A. M. de. Motivação e clima organizacional: um estudo de caso em uma Instituição Pública Federal de Ensino Superior do DF. Universitas Gestão e TI, Brasília, v. 6, n. 2, p. 1-15, jul./dez. 2016. Disponível em:

https://www.publicacoesacademicas.uniceub.br/gti/article/view/3877/3163. Acesso em: 5 mar. 2018. 
BEZERRA, F. A.; CORRAR, L. J. Utilização da análise fatorial na identificação dos principais indicadores para avaliação do desempenho financeiro: uma aplicação nas empresas de seguros. R. Cont. Fin., São Paulo: USP, n. 42, p. 50-62, set./dez. 2006.

BISPO, C. A. F. Um novo modelo de pesquisa de clima organizacional. Revista Produção, v. 16, n. 2, p. 258-273, maio/ago. 2006. Disponível em:

http://www.scielo.br/pdf/prod/v16n2/06.pdf. Acesso em: 05 mar. 2018.

CAMPBELL, D. T.; STANLEY, J. C. Delineamentos experimentais e quase experimentais de pesquisa. São Paulo: EPU, 1970.

CODA, R. Estudo sobre clima organizacional traz contribuição para aperfeiçoamento de pesquisa na área de RH. Revista de Administração, São Paulo. IA-USP, n. 75, dez. 1993.

CODA, R. Pesquisa de clima organizacional e gestão estratégica de recursos. In: BERGAMINI, C. W.; CODA, R. Psicodinâmica da vida organizacional da vida organizacional: motivação e liderança. São Paulo: Atlas, 1997.

CODA; R.; SILVA, D.; CUSTODIO, I, Multidimensional configurations of the organizational climate construct. The International Journal of Human Resource Management, 2014.

COSTA, A. L. A.; TANIGUCHI, K. Clima Organizacional: uma ferramenta estratégica para o diagnóstico em Recursos Humanos. Revista de Ciências Gerenciais, v. 13, n. 18, 2009. Disponível em: http://www.pgsskroton.com.br/seer/index.php/rcger/article/view/2620. Acesso em: 05 mar. 2018.

CURVO, L. D.; HEINZMANN, L. M. Estudo do clima organizacional da secretaria de gestão de pessoas de uma universidade federal. Revista Eletrônica Científica do CRA-PR, v. 4, n. 2, p. 1-18, 2017. Disponível em: http://recc.cra-pr.org.br/index.php/recc/article/view/72. Acesso em: 05 mar. 2018.

FIELD, A. Descobrindo a estatística usando o SPSS. 2. ed. Porto Alegre: Artmed, 2009.

FLICK, U. Introdução à metodologia da pesquisa: um guia para iniciantes. Porto Alegre: Penso, 2013.

FOREHAND, G. A.; GILMER, H. B. Environmental variation in studies of organizational behavior. Psychological Bulletin, v. 62, n. 6, p. 361-382, 1964.

GIL, A. C. Métodos e técnicas de pesquisa social. 6. ed. São Paulo: Atlas, 2008.

GRIFFIN, R. W.; MOORHEAD, G. Comportamento Organizacional Gestão de Pessoas e organizações. São Paulo: Cengage, 2016.

HAIR JR., J. F. et al. Análise multivariada de dados. 6. ed. Porto Alegre: Bookman, 2009. 
KINPARA, D. I.; LAROS, J. A. Clima Organizacional: Análise Fatorial Confirmatória de Modelos de Mensuração Concorrentes. Psic.: teor. e pesq., Brasília, v. 30, n. 1, p. 111-120, jan./mar. 2014. Disponível em: http://www.scielo.br/pdf/ptp/v30n1/14.pdf. Acesso em: 20 jun. 2017.

KOLB, D. A.; RUBIN, I. M.; McINTYRE, J. M. Psicologia organizacional: uma abordagem vivencial. São Paulo: Atlas, 1978.

LA FOLLETE, W. R.; SIMS, H. P. Is satisfaction redundant with organizational climate? Organizational, Stanford, 1975.

LIMA, L. C.; AMORIM, W. A. C. de; FISCHER, A. L. Da Racionalidade Instrumental para a Substantiva: Explorando Possibilidades da Gestão De Clima Organizacional. Teoria e prática em Administração, v. 5, n. 1, p. 159-182, 2015. Disponível em:

http://www.spell.org.br/documentos/ver/36651/da-racionalidade-instrumental-para-asubstantiva--explorando-possibilidades-da-gestao-de-clima-organizacional. Acesso em: 4 maio 2018.

LEWIN, K.; LIPPITT, R.; WHITE, R. K. Patterns of aggressive behavior in experimentally created "social climates". The Journal of Social Psychology, v. 10, p. 271-299, 1939.

LITWIN, G. H.; STRINGER, R. A. Motivation and organizational climate. Cambridge: Harvard University Press, 1968.

LUZ, R. S. Gestão do clima organizacional. Rio de janeiro: Qualitymark, 1995.

LUZ, R. S. Gestão do clima organizacional: proposta de critérios para metodologia de diagnóstico, mensuração e melhoria. Estudo de caso em organizações nacionais e multinacionais localizadas na cidade do rio de janeiro. 2003. 000f. Dissertação. (Mestrado em Sistemas de Gestão) - Programa de Pós-Graduação em Sistemas de Gestão, UFF, Niterói, 2003.

MALHOTRA, N. K. Pesquisa de marketing: foco na decisão. São Paulo: Pearson Prentice Hall, 2011.

MARTELO, M. R.; CLARO, J. A. C. S. ; VIEIRA, A.M. Clima Organizacional no contexto de uma incubadora: estudo com três empreendimentos do interior de São Paulo. Revista FSA, v. 13, n. 2, p.24-49, 2016.

MENEZES, I. G.; GOMES, A. C. P. Clima organizacional: uma revisão histórica do construto. Psicologia em Revista, v. 16, n.1, p. 158-179, 2010. Disponível em:

http://periodicos.pucminas.br/index.php/psicologiaemrevista/article/view/P.16789563.2010v16n1p158. Acesso em: 5 jul.2018.

MORAN, E. T.; VOLKWEIN, F. Examinando o clima organizacional em instituições de ensino superior. Pesquisa em Ensino Superior, v. 20, p. 367-383, 1988.

MORO, A. B. et al. Validação de um instrumento para medir o clima organizacional entre alunos de programas de pós-graduação de uma IES. Revista Eletrônica de Educação, v. 9, n. 
1 p. 181-195, 2015. Disponível em:

http://www.reveduc.ufscar.br/index.php/reveduc/article/viewFile/1007/387. Acesso em: 5 jul.2018.

NATÁRIO, M. M.; ARAÚJO, C.; COUTO, J. Higher Education Institution Organizational Climate Survey. International Journal of Advances in Management and Economics, v. 3, Issue 1, p. 107-121, jan./feb. 2014.

PEREIRA, I. L.; PEREIRA, E. L. O Clima Organizacional no Brasil: Pesquisa Bibliométrica nos Eventos da Anpad. Encontro da ANPAD, Rio de Janeiro, 38, 2014.

POCINHO, M. T. S. O livro que explica a estatística que precisa em 13 aulas IBM-SPSS \& Excel: teoria e prática. Coimbra: ESTSC/ISMT, 2014.

REGIS FILHO, G. I. Qualidade de vida no trabalho: o que as empresas precisam saber sobre ergonomia. Joinville: UNIVALI, 2015.

RIZZATTI, G. Análise de fatores significativos do clima organizacional da UFSC: contribuição para implantação do programa de qualidade. 1995. 237 f. Dissertação (Mestrado em Administração) - Programa de Pós-Graduação em Administração, Universidade Federal de Santa Catarina, Florianópolis, 1995. Disponível em:

https://repositorio.ufsc.br/handle/123456789/76386. Acesso em: 13 ago. 2018.

RIZZATTI, G. Categorias de análise de clima organizacional em universidades federais brasileiras. 2002. 307 f. Tese (Doutorado) - Programa de Pós-Graduação em Engenharia de Produção, Universidade Federal de Santa Catarina, 2018. Disponível em:

https://repositorio.ufsc.br/xmlui/handle/123456789/84206. Acesso em: 13 ago. 2018.

RODRIGUES, A. J. et al. Metodologia científica. Aracaju: UNIT, 2014.

SALDANHA, A. M. A atmosfera organizacional e o bem estar psíquico dos contribuintes individuais. São Paulo: Atlas, 1974.

SALGADO NETO, J. B. Clima organizacional da Uema: uma contribuição ao Programa "Uma Universidade para o Maranhão". 2001. Dissertação (Mestrado) - Programa de Pós-graduação em Administração, Universidade Federal de Santa Catarina, Florianópolis, 2001.

SAMPIERI, R. H.; COLLADO, C.F.; LUCIO, P.B. Metodologia de pesquisa. 3.ed. São Paulo: McGraw-Hill, 2013.

SANTOS, L. C.; VASQUEZ, O. C. A pesquisa de clima organizacional como instrumento de suporte à avaliação nas instituições de ensino superior. Avaliação, Campinas, v. 17, n. 1, p. $43-$ 63, 2012. Disponível em: http://www.scielo.br/pdf/aval/v17n1/a03v17n1.pdf. Acesso em: 15 ago. 2018.

SBRAGIA, R. Um estudo empírico sobre o clima organizacional em instituições de pesquisa.

Revista de Administração, São Paulo, v.18, n.2, p.30-39, abr./jun.1983. 
SCHNEIDER, B. Organizational climate: individual preference and organizational realities. Jounal of Applied Psychology, v. 56, p. 211-217, 1975.

SCHNEIDER, B.; BARBERA, K. (Orgs.) The Oxford Handbook of Organizational Climate and Culture. New York: Oxford University Press, 2014.

SCHNEIDER, B.; EHRHART, M. G.; MACEY, W. H. Organizational Climat and Culture. Annu. Rev. Psychol., v. 64, p. 361-88, 2013.

SILVA, N. Clima organizacional: uma proposta dos fatores a serem utilizados para avaliação do clima de uma Instituição de Ensino Superior. 2003. 140f. Dissertação (Mestrado) - Programa Pós-graduação em Engenharia de Produção, Universidade Federal de Santa Catarina, Florianópolis, 2003. Disponível em: https://repositorio.ufsc.br/handle/123456789/85479. Acesso em: 15 jun. 2018.

SOUZA, E. Clima e cultura organizacionais: como se manifestam e como se manejam SP: Edgard Blucher, 1978.

TACHIZAWA, T.; ANDRADE, R. O. Gestão de instituições de Ensino. Rio de Janeiro: FGV, 1999.

UNIVERSIDADE FEDERAL DE ALAGOAS. 2018. Disponível em: https://ufal.br/. Acesso em: 5 jul. 2018.

UNIVERSIDADE FEDERAL DE ALAGOAS. Plano anual de capacitação: UFAL/2016. Maceió: UFAL, 2016.

UNIVERSIDADE FEDERAL DE ALAGOAS. Plano anual de capacitação: UFAL/2017. Maceió: UFAL, 2017.

UNIVERSIDADE FEDERAL DE ALAGOAS. Plano anual de capacitação: UFAL/2018. Maceió: UFAL, 2018.

UNIVERSIDADE FEDERAL DE ALAGOAS. Plano de desenvolvimento institucional: 2013-2017. Maceió: UFAL, 2013.

UNIVERSIDADE FEDERAL DE ALAGOAS. Prestação de contas ordinárias anual: relatório de gestão. Exercício 2017. Maceió: UFAL, 2018.

UNIVERSIDADE FEDERAL DE ALAGOAS. Relatório de autoavaliação: UFAL/2016. Maceió: UFAL, 2017.

VELA, H. H.; GARRIDO, N. P.; PÉREZ, C. R. Diseño De Instrumento Para evaluar clima organizacional de universidades públicas en México. European Scientific Journal, v. 12, n. 28, Oct. 2016. 
VIEIRA, S. Introdução à bioestatística. Rio de Janeiro: Elsevier, 2011.

ZAMBRANO CAMPOVERDE, J.; RAMÓN PINEDA, M. Á.; ESPINOZA-FREIRE, E. Estudio sobre el clima organizacional en los docentes de la Universidad Técnica de Machala. Universidad y Sociedad, v. 9, n. 2, p.163-172, 2017. 